\title{
Adaptive Channel Estimation in OFDM System Using Cyclic Prefix (Kalman Filter Approach)
}

\author{
P. V. NAGANJANEYULU ${ }^{1}$, K. SATYA PRASAD ${ }^{2}$ \\ ${ }^{1}$ Department of ECE, Guntur Engineering College, Guntur, India \\ ${ }^{2} J N T U$, Kakinada, India \\ E-mail: \{pvnaganjaneyulu,prasad_kodati\}@yahoo.co.in \\ Received August 31, 2009; revised October 1, 2009; accepted October 29, 2009
}

\begin{abstract}
OFDM is a promising technique for high data rate transmission and the channel estimation is very important for implementation of OFDM. In this paper, cyclic prefix (CP) can be used as a source of channel information which is originally used to reduce inter symbol interference (ISI). Based on this CP observation, we propose two cross coupled dual Kalman filters to track the channel variations without additional training sequences. One Kalman filter AR parameter estimation and another for fading channel estimation.
\end{abstract}

Keywords: Cyclic Prefix, Kalman Filter

\section{Introduction}

In OFDM systems, due to user mobility, each carrier is subject to Doppler shifts resulting in time-varying fading. Thus, the estimation of the fading process over each carrier is essential to achieve coherent symbol detection at the receiver. In that case, training sequence/pilot aided techniques and blind techniques are two basic families for channel estimation. Training based methods require the transmission of explicit pilot sequences followed by suitable filtering. This paper focuses on estimation of fading wireless channels for OFDM, using the ideas of Cyclic Prefix (CP) based estimation and adaptive filtering.

The time-varying fading channels are usually modelled as zero-mean wide-sense stationary circular complex Gaussian processes, whose stochastic properties depend on the maximum Doppler frequency denoted by $f_{d}$. According to the Jakes model [1], the theoretical Power Spectrum Density (PSD) of the fading process, is band-limited. Moreover, it exhibits twin peaks at $\pm f_{d}$. The fading wireless channel statistics can be directly estimated by means of the Least Mean Square (LMS) and the Recursive Least Square (RLS) algorithms as in [2]. Alternatively, Kalman filtering algorithm combined with an Autoregressive (AR) model to describe the time evolution of the fading processes and it provides superior performance over the LMS and RLS based channel estimators in [3]. In addition, when the AR model parameters are unknown, dual filtering algorithms are used to estimate the fading channels.
In this paper, for the channel estimation of OFDM, a system model and architecture over fading channels are presented. In the next section a CP based model and the different channel estimation algorithms Kalman and Dual-Kalman are discussed. The performance results are discussed in the next section, finally simulation results are presented.

\subsection{Existing Methods for Channel Estimation}

Different Channel Estimation methods are proposed based on training sequence, blind and semi-blind. In practice we either assume the channel is invariant and use the initial training to get the channel estimation are periodically employ training sequence to trap the channel variations. These will cause performance loss or increase the overhead of the system. So, we present that the CP in OFDM which is used to reduce ISI and normally discarded at the receiver can be viewed as a training sequence for channel estimation. In paper [3], channel estimation is proposed by two Kalman filters based on noisy data as training sequence. In this paper, we present two cross coupled Kalman by using CP as training sequence and their performances are compared.

\section{System Model}

In the following, we consider a low to moderate Doppler environment, which allows for a block fading (quasistatic) channel assumption. This implies that the channel 


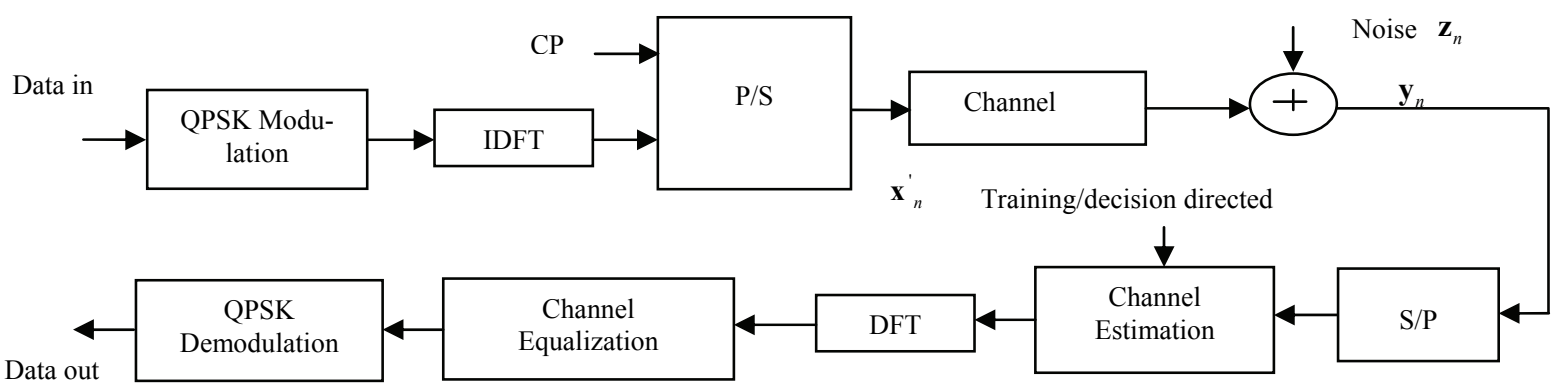

Figure 1. Block schematic of the OFDM system.

tap variations within OFDM symbol duration are negligible, and hence we may define an $L \times 1$ channel tap vector for each OFDM symbol as

$$
\mathbf{h}_{n}=\left[h_{n}(0) h_{n}(1) \ldots h_{n}(L-1)\right]^{T}
$$

where $h_{n}(l)$ is the $l^{\text {th }}$ channel tap for the $n^{\text {th }}$ OFDM symbol.

The classical Doppler spectrum for each of the $L$ channel taps is approximated by an independent AR-2 process [4].

For the $l^{\text {th }}$ channel tap at $n^{\text {th }}$ OFDM symbol, we have

$$
h_{n}(l)=-a_{1} h_{n-1}(l)-a_{2} h_{n-2}(l)+v_{n}(l)
$$

where $a_{1}$ and $a_{2}$ are the AR-2 coefficients are defined in [5] and $v_{n}(l)$ is the modelling noise for $l^{\text {th }}$ tap at symbol $n$.

\subsection{OFDM Architecture over Fading Channel}

We consider an OFDM system as in Figure 1 with $N$ data subcarriers. Input data are buffered, converted to a parallel stream and modulated to i.i.d. equi-probable symbols $X_{n}(k)$, where $X_{n}(k)$ denotes the $k^{\text {th }}$ symbol of the $n^{\text {th }}$ OFDM symbol. Each symbol mapped to some complex constellation points, $X_{n}(k), k=0,1, \ldots, N-1$ at each $n$. The modulation is implemented by $N$-point inverse discrete Fourier transform (IDFT) for the symbol vector

$$
\mathbf{X}_{n}=\left[X_{n}(0) X_{n}(1) \ldots X_{n}(N-1)\right]^{T}
$$

is

$x_{n}(m+g i)=\frac{1}{\sqrt{N}} \sum_{k=0}^{N-1} X_{n}(k) e^{j 2 \pi m k / N}, 0 \leq \mathrm{m} \leq N-1 \mathrm{CP}$ of length $g i$ is appended to form the transmitted vector as

$$
\mathbf{x}_{n}^{\prime}=\left[x_{n}(0) x_{n}(1) \ldots x_{n}(g i-1): x_{n}(g i) x_{n}(g i+1) \ldots x_{n}(g i+N-1)\right]^{T}(4)
$$

where

$$
x_{n}(m)=x_{n}(N+m), \quad 0 \leq m \leq g i-1
$$

The received symbol corrupted by fading channel and AWGN becomes

$$
y_{n}(m)=\sum_{l=0}^{L-1} h_{n}(l) x_{n}(m-l)+z_{n}(m), 0 \leq \mathrm{m} \leq N+g i+L-1
$$

where $n$ is the OFDM symbol index,

$z n(m)$ is an AWGN sample with zero mean and variance $\sigma^{2}$ at instant $m$ in the $n^{\text {th }}$ OFDM symbol.

Demodulation involves removing the cyclic prefix and taking $N$-point DFT of the received vector to get

$$
\mathbf{Y}_{n}=\left[Y_{n}(0) \mathrm{Y}_{n}(1) \ldots \mathrm{Y}_{n}(N-1)\right]^{T}
$$

In frequency domain, we have over each subcarrier

$$
Y_{n}(k)=X_{n}(k) H_{n}(k)+Z_{n}(k)
$$

where $H_{n}(k)$ is the channel frequency response at subcarrier $k$ given by

$$
H_{n}(k)=\frac{1}{\sqrt{N}} \sum_{l=0}^{L-1} h_{n}(l) e^{-j 2 \pi l k / N}, 0 \leq k \leq N-1
$$

and $z_{n}(k)$ is the noise on $k^{\text {th }}$ subcarrier of $n^{\text {th }}$ OFDM symbol i.e.,

$$
Z_{n}(k)=\frac{1}{\sqrt{N}} \sum_{m=0}^{N-1} z_{n}(m) e^{-j 2 \pi m k / N}, 0 \leq k \leq N-1
$$

At the receiver, the channel estimator is followed by frequency domain equalizer. After equalization, the estimated symbol at the $k^{\text {th }}$ symbol becomes

$$
\hat{X}_{n}(k)=\frac{Y_{n}(k)}{\hat{H}_{n}(k)}=\frac{X_{n}(k) H_{n}(k)}{\hat{H}_{n}(k)}+\frac{Z_{n}(k)}{\hat{H}_{n}(k)}
$$

where $\hat{H}_{n}(k)$ is the estimate of $H_{n}(k)$ defined in Equation (8). The estimated symbols $\hat{X}_{n}(k)$ are then demapped to output bits.

\section{CP Based Channel Estimation Techniques}

This section describes the use of various adaptive filtering algorithms in CP based frame work for channel estimation in OFDM systems. From Equation (5), we know that

$$
y_{n}(m)=h_{n}(0) x(m)+h_{n}(1) x(m-1)+\ldots+h_{n}(L-1) x(m-L+1)+z_{n}(m)(
$$

Gathering the received samples of the $n^{\text {th }}$ received OFDM symbol for time instants $0 \leq m \leq g i-1$, we obtain a $g i \times 1$ vector

$$
\mathbf{y}_{n, C P}=\left[y_{n}(0) y_{n}(1) y_{n}(2) \ldots y_{n}(g i-1)\right]^{T}
$$


which is the $\mathrm{CP}$ of the received OFDM symbol, and

$$
\mathbf{z}_{n, C P}=\left[z_{n}(0) z_{n}(1) z_{n}(2) \ldots z_{n}(g i-1)\right]^{T}
$$

is the $g i \times 1$ vector of AWGN samples affecting the CP part of the $n^{\text {th }}$ received OFDM symbol.

\subsection{Kalman Filtering (KF) Algorithm}

When operating in a non-stationary environment, Kalman filter [6] is known to yield an optimal solution to the linear filter problem. This subsection describes the application of KF to the channel estimation problem in OFDM. For this purpose, the system is formulated as a state-space model, with unknown channel taps comprising the state of the system. We assume that the state $\mathbf{S}_{\mathrm{n}}$, to be estimated at OFDM symbol index $n$, comprises of channel taps at two consecutive OFDM symbols [7].

$$
\mathbf{s}_{n}=\left[\mathbf{h}_{n-1} \mathbf{h}_{n}\right]_{2 L \times 1}^{T}
$$

From Equation (1) and Equation (2) we have

$$
\begin{gathered}
\mathbf{h}_{n}=\left[h_{n}(0) h_{n}(1) \ldots h_{n}(L-1)\right]_{L \times 1}^{T} \\
y_{n}(m)=h_{n}(0) x(m)+h_{n}(1) x(m-1)+\ldots+h_{n}(L \\
{\left[\begin{array}{c}
y_{n}(0) \\
y_{n}(1) \\
\vdots \\
y_{n}(g i-1)
\end{array}\right]=\left[\begin{array}{ccc}
x_{n}(0) & x_{n-1}(N+g i-1) & \ldots \\
x_{n}(1) & x_{n}(0) & x_{n-1}(N+g i-1) \\
\vdots & \vdots & \vdots \\
x_{n}(g i-1) & x_{n}(g i-2) & \ldots
\end{array}\right.}
\end{gathered}
$$$$
y_{n}(m)=h_{n}(0) x(m)+h_{n}(1) x(m-1)+\ldots+h_{n}(L-1) x(m-L+1)+z_{n}(m)
$$

$$
\mathbf{h}_{n-1}=\left[h_{n-1}(0) h_{n-1}(1) \ldots h_{n-1}(L-1)\right]_{L \times 1}^{T}
$$

and

$$
\mathbf{h}_{n}=a_{1} \mathbf{h}_{n-1}+a_{2} \mathbf{h}_{n-2}+\mathbf{v}_{n}
$$

From above equations we get

$$
\left[\begin{array}{l}
\mathbf{h}_{n-1} \\
\mathbf{h}_{n}
\end{array}\right]=\left[\begin{array}{cc}
\mathbf{0}_{L \times L} & \mathbf{I}_{L} \\
a_{2} \mathbf{I}_{L} & a_{l} \mathbf{I}_{L}
\end{array}\right]\left[\begin{array}{l}
\mathbf{h}_{n-2} \\
\mathbf{h}_{n-1}
\end{array}\right]+\mathbf{v}_{n}-
$$

We observe that Equation (16) provides the basis for forming the process equation as

$$
\mathbf{s}_{n}=\mathbf{B} \mathbf{s}_{n-1}+\mathbf{v}_{n}
$$

Here, transition matrix

$$
\mathbf{B}=\left[\begin{array}{ll}
\mathbf{0}_{L \times L} & \mathbf{I}_{L} \\
a_{2} \mathbf{I}_{L} & a_{l} \mathbf{I}_{L}
\end{array}\right]_{2 L \times 2 L}
$$

$\mathbf{0}_{L \times L}$ denotes the $L \times L$ matrix of all zeros and $\mathbf{I}_{L}$ is the $L \times L$ identity matrix.

Process noise vector

$$
\mathbf{v}_{n}=\left[\mathbf{0}_{1 \times \mathrm{L}} \vdots v_{n}(0) v_{n}(1) \ldots v_{n}(L-1)\right]_{2 L \times 1}^{T}
$$

where $v_{n}(l)$ is the modelling noise as in (2)

From Equation (11), we have where $0 \leq m \leq g i-1$

We observe from above that following provides the basis for forming measurement equation as

$$
\mathbf{y}_{n, C P}=\overline{\mathbf{A}}_{n} \mathbf{s}_{n}+\mathbf{z}_{n, C P}
$$

where the measurement matrix $\overline{\mathbf{A}}_{n}$ in Equation (20) is formed from the matrix $\mathbf{A}_{n}$ by augmenting it with a null matrix as

$$
\overline{\mathbf{A}}_{n}=\left[\begin{array}{lll}
\mathbf{0}_{g i \times \mathrm{L}} & \vdots & \mathbf{A}_{n}
\end{array}\right]_{g i \times 2 L}
$$

Here $\mathbf{A}_{n}$ is a $g i \times L$ matrix of transmitted symbols that determine the CP of the received OFDM symbol.

$$
\mathbf{A}_{n}=\left[\begin{array}{cccc}
x_{n}(0) & x_{n-1}(N+g i-1) & \ldots & x_{n-1}(N+g i-L+1) \\
x_{n}(1) & x_{n}(0) & x_{n-1}(N+g i-1) & \ldots \\
\vdots & \vdots & \vdots & \vdots \\
x_{n}(g i-1) & x_{n}(g i-2) & \ldots & x_{n}(g i-L)
\end{array}\right]_{g i \times L}
$$

Considering that the CP appended to an OFDM symbol is a replication of the last gi values of that symbol, we may write $\mathbf{A}_{n}$ in terms of transmitted $\mathrm{CP}$ value as,

$$
\mathbf{A}_{n}=\left[\begin{array}{ccccc}
x_{n}(0) & x_{n-1}(g i-1) & x_{n-1}(g i-2) & \ldots & x_{n-1}(g i-L+1) \\
x_{n}(1) & x_{n}(0) & x_{n-1}(g i-1) & \ldots & x_{n-1}(g i-L+2) \\
\vdots & \vdots & \vdots & & \vdots \\
x_{n}(g i-1) & x_{n}(g i-2) & \ldots & \ldots & x_{n}(g i-L)
\end{array}\right]_{g i \times L}
$$

$\mathbf{A}_{n}$ has gi rows corresponding to gi consecutive time instants of the CP. The $L$ elements of each row are the transmitted symbol values affecting the received $\mathrm{CP}$ value at that instant. This matrix structure assumes that the $\mathrm{CP}$ length is at least equal to the number of taps in the channel impulse response, i.e. no inter block interference.

The measurement noise vector $\mathbf{Z}_{n, C P}$, in Equation (20), comprises the gi $\times 1$ vector of AWGN samples affecting the cyclic prefix part of the OFDM symbol.

We observe that Equation (17) and Equation (20) provide the basis for forming the process equation and measurement equation, respectively for the state space model, as follows

$$
\begin{gathered}
\mathbf{s}_{n}=\mathbf{B s}_{n-1}+\mathbf{v}_{n} \\
\mathbf{y}_{n, C P}=\overline{\mathbf{A}}_{n} \mathbf{s}_{n}+\mathbf{z}_{n, C P}
\end{gathered}
$$

A Kalman filter is employed to estimate the unknown 
state of the system. Cyclic prefix of the received OFDM symbol $\mathbf{y}_{n, C P}$ is given as input observation to Kalman algorithm, the following estimation equations are given by [3]

$$
\begin{gathered}
{\left[\boldsymbol{P}_{n \mid n-1}\right]_{2 L \times 2 L}=\mathbf{B} \boldsymbol{P}_{n-1 \mid n-1} \mathbf{B}^{H}+\mathbf{Q}_{1}} \\
{\left[\boldsymbol{\alpha}_{n}\right]_{g i \times 1}=\left[\mathbf{y}_{n, C P}-\overline{\mathbf{A}}_{n} \hat{\mathbf{s}}_{n-1}\right]} \\
{\left[\mathbf{C}_{n}\right]_{g i \times g i}=\overline{\mathbf{A}}_{n} \boldsymbol{P}_{n \mid n-1} \overline{\mathbf{A}}_{n}^{H}+\mathbf{Q}_{2}} \\
{\left[\mathbf{K}_{n}\right]_{2 L \times g i}=\boldsymbol{P}_{n \mid n-1} \overline{\mathbf{A}}_{n}^{H} \mathbf{C}_{n}^{-1}} \\
{\left[\hat{\mathbf{s}}_{n}\right]_{2 L \times 1}=\mathbf{B} \hat{\mathbf{s}}_{n-1}+\mathbf{K}_{n} \boldsymbol{\alpha}_{n}} \\
{\left[\hat{\mathbf{h}}_{n}\right]_{L \times 1}=R \hat{\mathbf{s}}_{n}, \quad R=\left[\mathbf{0}_{L \times L} \mathbf{I}_{L}\right]_{L \times 2 L}} \\
{\left[\boldsymbol{P}_{n}\right]_{2 L \times 2 L}=\left[\mathbf{I}_{2 L}-\mathbf{K}_{n} \overline{\mathbf{A}}_{n}\right] \boldsymbol{P}_{n \mid n-1}}
\end{gathered}
$$

where $\mathbf{K}_{n}$ is the $2 L \times g i$ Kalman gain matrix, $\hat{\mathbf{s}}_{n}$ is the state estimate at the $n^{\text {th }}$ OFDM symbol, $\mathbf{Q}_{1}$ and $\mathbf{Q}_{2}$ are the covariance matrices of $\mathbf{v}_{n}$ and $\mathbf{Z}_{n, C P}$ respectively, $\boldsymbol{P}_{n \mid n-1}$ is the priori covariance matrix of estimation error, and $\boldsymbol{P}_{n}$ is the current covariance matrix of estimation error. When the channel taps are modelled as a zero mean random process, the algorithm is initialized with an all-zero state vector. Besides this, the assumption of uncorrelated scattering (US) causes the different channel taps to be i.i.d., and the error covariance matrix is initialized as an identity matrix.

$$
\begin{gathered}
\mathbf{s}_{0}=\hat{\mathbf{s}}_{0}=\mathbf{0}_{2 L \times 1} \\
\boldsymbol{P}_{0}=E\left[\left(\mathbf{s}_{0}-\hat{\mathbf{s}}_{0}\right)\left(\mathbf{s}_{0}-\hat{\mathbf{s}}_{0}\right)^{H}\right]=\mathbf{I}_{n}
\end{gathered}
$$

The receiver operates in training and decision directed modes. In training mode the known transmitted $\mathrm{CP}$ $\left(\mathbf{x}_{n, C P}\right)$ and CP part of the received OFDM symbol $\left(\mathbf{y}_{n, C P}\right)$ form the input to the above Kalman filter algorithm, and get the channel estimation $H_{n}(k)$, we get

$$
\hat{X}_{n}(k)=\frac{Y_{n}(k)}{\hat{H}_{n}(k)}
$$

In decision directed mode the receiver uses the estimated channel vector from the previous OFDM symbol to demodulate the received symbol and generate an estimate of transmitted CP $\left(\hat{X}_{n, C P}(k)\right)$. Here the transmitted CP part can be estimated by previous estimated channel i.e.,

$$
\hat{X}_{n, C P}(k)=\frac{Y_{n, C P}(k)}{\hat{H}_{n-1}(k)}
$$

This estimated CP and CP of the received OFDM symbol $\left(\mathbf{y}_{n, C P}\right)$ helps to estimate the channel.

The equations from (23) to (29) can be carried out by providing the AR parameters that are involved in the transition matrix $\mathbf{B}$ and the driving process variances are available. In case, these are unknown, for estimating these parameters Dual-Kalman filtering technique is used.

\subsection{Dual-Kalman Filtering Algorithm}

To estimate the AR parameters $\boldsymbol{\theta}_{n}$ from the estimated fading process $\hat{\mathbf{h}}_{n}$, Equation (28) is firstly represented as an AR-2 model to express the estimated fading process as a function of $\boldsymbol{\theta}_{n}$ (AR parameter vector).

$$
\begin{aligned}
& \hat{\mathbf{h}}_{n}=\left[\begin{array}{ll}
\hat{\mathbf{h}}_{n-1} & \hat{\mathbf{h}}_{n-2}
\end{array}\right]\left[\begin{array}{l}
a_{1} \\
a_{2}
\end{array}\right]+\mathbf{w}_{n}
\end{aligned}
$$

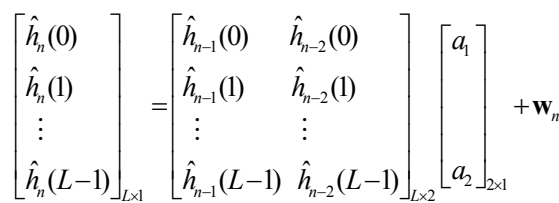

$$
\begin{aligned}
& \mathbf{r}_{n}=\mathbf{H} \boldsymbol{\theta}_{n}+\mathbf{w}_{n}
\end{aligned}
$$

where $\mathbf{r}_{\boldsymbol{n}}$ is the estimated channel vector, $\boldsymbol{\theta}_{\boldsymbol{n}}$ is the AR parameter vector defines as $\boldsymbol{\theta}_{n}=\left[\begin{array}{ll}a_{1} & a_{2}\end{array}\right]^{T}$.

and $\mathbf{w}_{\boldsymbol{n}}$ is the $L \times 1$ noise vector as in Equation (19).

When the channel is assumed to be stationary, the AR parameters are time-invariant and satisfy the following relationship

$$
\boldsymbol{\theta}_{n}=\boldsymbol{\theta}_{n-1}
$$

As Equation (33) and Equation (34) define a statespace representation for the estimation of the AR parameters, a second Kalman filter can be used to recursively estimate $\boldsymbol{\theta}_{\boldsymbol{n}}$ as follows [3]

$$
\begin{gathered}
{\left[\boldsymbol{P}_{\theta_{n / n-1}}\right]_{2 \times 2}=\boldsymbol{P}_{\theta_{n-1 / n-1}}} \\
{\left[\boldsymbol{\alpha}_{\theta_{n}}\right]_{L \times 1}=\left[\mathbf{r}_{n}-\mathbf{H} \hat{\boldsymbol{\theta}}_{n-1}\right]} \\
{\left[\mathbf{C}_{\theta_{n}}\right]_{L \times L}=\mathbf{H} \boldsymbol{P}_{\theta_{n / n-1}} \mathbf{H}^{H}+\mathbf{Q}_{3}} \\
{\left[\mathbf{K}_{\theta_{n}}\right]_{2 \times L}=\boldsymbol{P}_{\theta_{n / n-1}} \mathbf{H}^{H} \mathbf{C}_{\theta_{n}}{ }^{-1}} \\
{\left[\hat{\boldsymbol{\theta}}_{n}\right]_{2 \times 1}=\hat{\boldsymbol{\theta}}_{n-1}+\mathbf{K}_{\theta_{n}} \boldsymbol{\alpha}_{\theta_{n}}} \\
{\left[\boldsymbol{P}_{\theta_{n / n}}\right]_{2 \times 2}=\left[\mathbf{I}_{2}-\mathbf{K}_{\theta_{n}} \mathbf{H}\right] \boldsymbol{P}_{\theta_{n / n-1}}}
\end{gathered}
$$

where $\mathbf{Q}_{3}$ is the covariance matrix of the $\mathbf{w}_{n}$, the error covariance matrix and the initial AR parameter vector are defined as

$$
\begin{gathered}
\hat{\boldsymbol{\theta}}_{0}=\boldsymbol{\theta}_{0}=\mathbf{0}_{2 \times 1} \\
\boldsymbol{P}_{\theta_{0 / 0}}=E\left[\left(\boldsymbol{\theta}_{0}-\hat{\boldsymbol{\theta}}_{0}\right)\left(\boldsymbol{\theta}_{0}-\hat{\boldsymbol{\theta}}_{0}\right)^{H}\right]=\mathbf{I}_{2}
\end{gathered}
$$

\subsection{Noise parameters estimation}

Apart from estimating the AR parameters, we also need to estimate the noise parameters for the fading channel 


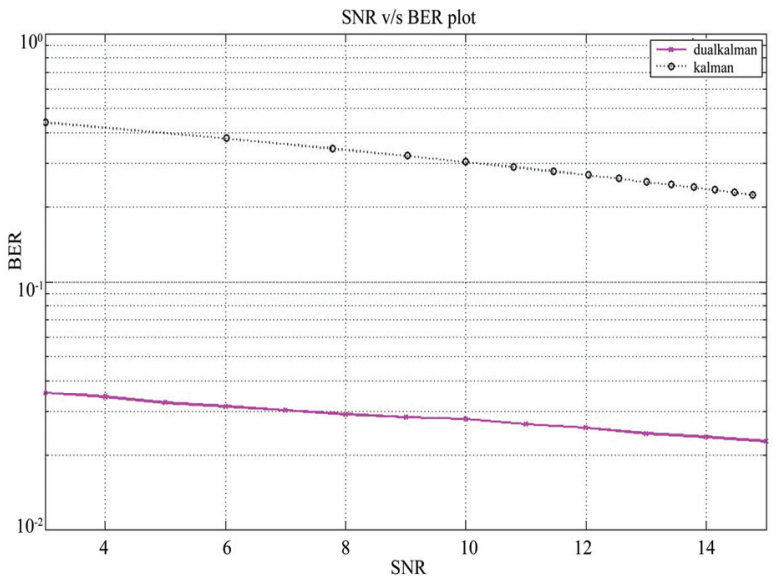

Figure 2. BER v/s SNR for Kalman and Dual Kalman.

environment i.e., variance of $\mathbf{v}_{n}$ and $\mathbf{Z}_{n, C P}$. This can be done by using the error covariance matrices. From Equation (23) and Equation (29) we can write the noise variances recursively as

$$
\begin{gathered}
{\left[\boldsymbol{L}_{n}\right]_{2 L \times 2 L}=\boldsymbol{P}_{n}-\mathbf{B} \boldsymbol{P}_{n-1 \mid n-1} \mathbf{B}^{H}+\mathbf{K}_{n} \boldsymbol{\alpha}_{n} \boldsymbol{\alpha}_{n}^{H} \mathbf{K}_{n}^{H}} \\
\hat{Q}_{1}(n)=\frac{n-1}{n} \hat{Q}_{1}(n-1)+\frac{1}{n} \boldsymbol{D} \boldsymbol{L}_{n} \boldsymbol{D}^{T} \\
\boldsymbol{D}=[10 \ldots . .0]_{1 \times 2 L} \\
{\left[\mathbf{M}_{n}\right]_{g i \times g i}=\boldsymbol{\alpha}_{n} \boldsymbol{\alpha}_{n}^{H}-\overline{\mathbf{A}}_{n} \boldsymbol{P}_{n \mid n-1} \overline{\mathbf{A}}_{n}^{H}} \\
\hat{Q}_{2}(n)=\frac{n-1}{n} \hat{Q}_{2}(n-1)+\frac{1}{n} \boldsymbol{D}_{1} \mathbf{M}_{n} \boldsymbol{D}_{1}^{T} \\
\boldsymbol{D}_{1}=[10 \ldots . .0]_{1 \times g i},
\end{gathered}
$$

where $\hat{Q}_{1}(n)$ and $\hat{Q}_{2}(n)$ are the estimated variances of the process noise $\mathbf{v}_{n}$ and modelling noise $\mathbf{Z}_{n, C P}$ respectively.

\section{Results and Conclusions}

In this analysis we compare $\mathrm{CP}$ based dual Kalman and Kalman. At SNR of 10db, BER of Kalman is $10^{-0.8}$ where as for dual Kalman; this value is $10^{-1.81}$. For Overall SNR is concerned, dual Kalman gives better performance and doesn't require any additional training sequence like training bits and noise. (Figure 2)

\section{References}

[1] R. Steele, "Mobile radio communications," New York: IEEE Press, 1992.

[2] X. W. Wang and K. J. R. Liu, "Adaptive channel estimation using cyclic prefix in multicarrier system," IEEE Communication Letters Magazines, Vol. 3, pp. 291-293, October 1999.

[3] A. Jamoos, D. Labarre, E. Grivel, and Najim, "Two cross coupled Kalman filters for joint estimation of MC-DSCDMA fading channels and their corresponding autoregressive parameters," Proceedings of EUSIPCO, Antalya, Turkey, September 4-8, 2005.

[4] M. K. Tsatsanis, G. B. Giannakis, and G. Zhou, "Estimation and equalization of fading channels with random coefficients," Signal Process, Vol. 53, pp. 211-229, 1996.

[5] A. Jamoos, J. Grolleau, and E. Grivel, "Kalman vs $\mathrm{H}_{\infty}$ algorithms for MC-DS-CDMA channel estimation with or without a priori AR modelling," IEEE Multicarrier Spread Spectrum, Springer Verlag, pp. 427-436, 2007.

[6] I. R. Petersen and A. V. Savkin, "Robust Kalman filtering for signals and systems with large uncertainties," Boston, MA: Birkhäuser, 1999.

[7] Y. Li, L. J. Cimini, and N. R. Sollenberger, "Robust channel estimation for OFDM systems with rapid dispersive fading channels," IEEE Transactions on Communications, Vol. 46, pp. 902-915, July 1998. 